\section{Evaluation of dental practitioners with special interest in minor oral surgery}

\author{
A. Pau, ${ }^{1}$ S. Nanjappa ${ }^{2}$ and S. Diu ${ }^{3}$
}
IN BRIEF
- Indicates that a minor oral surgery service provided in the primary care setting is acceptable to patients.
- A primary care minor oral surgery service is cost-effective compared to treatment under a secondary care setting.
- A primary care minor oral surgery service supports local dentists in their management of patients requiring minor oral surgery.

This paper reports an evaluation of dental practitioners with special interest (DPwSI) in minor oral surgery (MOS). Objectives To: i) audit the appropriateness of referrals to a DPwSI-MOS service, ii) determine its cost-effectiveness, iii) gather the views of patients who had been treated, and iv) assess the referring dentists' views. Methods Analysis of management information, telephone interviews with patients, postal questionnaire survey of referring dentist. Results $0 f 83$ referrals received, 51 (five for consultation and 46 for extractions) were seen by a DPwSI. The average waiting time between the referral and the treatment dates was 36 days. The cost of treating 51 cases was $£ 8,020$, compared to $£ 43,608$ under secondary care, a difference of $€ 35,588$ and a projected annual difference of $€ 142,352$. Eighteen patients were interviewed after attending for treatment. Most reported that they were seen within two weeks (ten, 57\%) and four weeks (five, 28\%) from the time they were referred. The majority $(15,83 \%)$ rated the service as good to excellent. Of the questionnaires mailed to 90 referring dentists, 45 (50\%) were returned. Of the 40 (90\%) dentists who reported being aware of the DPwSIMOS, 24 (60\%) reported having used the service. Conclusions This pilot has demonstrated that the provision of MOS in the primary care setting by DPwSIs can provide a good service for patients, potentially improve access for patients, support patient management for referring dentists and reduce costs for the PCT.

\section{INTRODUCTION}

Since 1 April 2006, primary care trusts (PCTs) have been charged with the legal duty of commissioning primary dental services to reflect local needs and priorities. ${ }^{1}$ Through this legislation the Government aims to fulfil its commitment to ensuring access to safe and effective dental care with equitable outcomes, with the tenet of value for money underpinning such commissioning. This commitment was reinforced by the $11 \%$ increase in dental funding allocated to PCTs for 2008/09, who are expected to demonstrate that they are making effective use of the additional funding and delivering year

${ }^{1}$ Specialist Registrar/Honorary Senior Lecturer in Dental Public Health, Dental Institute, King's College London, Caldecot Road, Denmark Hill, London SE5 9RW: ${ }^{2} \mathrm{PhD}$ Candidate, Institute of Dentistry, Barts and The London, Queen Mary University of London, Turner Street, London E1 2AD; ${ }^{3}$ Consultant in Dental Public Health, Havering PCT, St. George's Hospital, Suttons View, 117 Suttons Lane, Hornchurch, Essex RM12 6RS ${ }^{*}$ Correspondence to: Dr Allan Pau Email:allan.pau@kcl.ac.uk

\section{Refereed Paper}

Accepted 24 November 2009

DOI: $10.1038 /$ sj.bdj.2010.101

${ }^{\circledR}$ British Dental Journal 2010; 208: 103-107 on year improvements in the number of patients accessing local dental services. ${ }^{2}$

An area in which access may be improved is specialised-type dental services, such as minor oral surgery (MOS), orthodontics and dental treatment under sedation. Most of these services are currently provided in secondary care settings which people may find difficult to access because of transport problems or physical immobility. Furthermore the provision of such services in secondary care settings place considerable financial constraints on the budgets of PCTs. The concept of health practitioners with special interests is a service reform that was first introduced in the NHS Plan to allow for the provision of a range of specialist-type care procedures within a primary care setting, thus making them more locally accessible. ${ }^{3}$

Dental practitioners with a special interest (DPwSIs) are dental practitioners working in a primary care environment, who provide special interest services in addition to having a generalist role. ${ }^{4}$ DPwSIs are endorsed and supported by the Department of Health and the Faculty of General Dental Practitioners (UK), who consider them as means of delivering safe and high quality care in local and convenient settings through using appropriate skills mix, and encouraging dentists to develop their practice within the NHS. Examples of such services include DPwSI in minor oral surgery (MOS), sedation, orthodontics, special needs, endodontics and periodontics. The advent of DPwSIs allows PCTs to contract general dental practitioners who have developed special interests where there is a local need. In addition to facilitating PCT commissioning services with the best value for money, DPwSIs are also means for tackling hospital waiting time and meeting the 18-week patient pathway as outlined in the NHS Improvement Plan. ${ }^{5}$

In light of data from the London Health Observatory reporting that in 2005/06 Havering PCT had the highest number of wisdom teeth extractions per 100,000 population, at 170 cases compared to an average of 62 for London as a whole, ${ }^{6}$ the decision was taken to explore using DPwSIs in MOS to manage wisdom teeth extractions in Havering PCT. Minor oral surgery for Havering PCT residents is usually provided by Barking, Havering and Redbridge 
NHS Trust (BHRT), which includes Barking Hospital, King George Hospital, Queen's Hospital and Victoria Hospital (Romford). A three-month project to pilot the provision of minor oral surgical extractions by DPwSIs in the primary dental care setting was implemented on 14 May 2007. To date, there has been local commissioning of DPwSIs across England, but the overall provision is scant.7 This paper describes the pilot project and reports its evaluation, with a view to sharing good practice and promoting DPwSIs as facilitating a care pathway to improve local access to specialist-type services. The aims are to: i) audit the referral process and cost-effectiveness of the project, ii) gather views of patients who had received treatment, and iii) assess primary care dentists' views of the project.

\section{METHODS}

A project team, comprising the dental services manager, clinical assessment service manager, dental practice advisor, a dental public health consultant and a maxillofacial surgery consultant was convened. The team studied BHRT's waiting list for the types of dental procedures that had been referred in and proposed that DPwSIMOS should initially be intended for wisdom teeth extraction and extractions of special difficulty such as retained roots. A letter was mailed to all Havering PCT dentists informing them of the proposed service and inviting them to express their interest in becoming a DPwSI-MOS. Each expression of interest was required to be accompanied by a portfolio of evidence demonstrating oral surgery experience, and a reference from a maxillofacial consultant confirming post-graduation experience in oral surgery. They were also asked for their feedback on a draft referral form. Three DPwSIs were appointed following interviews by the team.

Referring dentists were informed of the service by post together with instructions for referring patients who required extractions of special difficulty to the Clinical Assessment Service (CAS) using a referral form provided by the PCT. Relevant radiographs were attached with each referral and mailed to the CAS. All referrals were assessed by a PCT appointed dental clinician. The outcomes of this assessment were one of the following:
- Referral forwarded to one of three appointed DPwSIs, usually the one nearest to where the patient resided

- Referral forwarded to the Oral and Maxillofacial Surgery Department at Queen's Hospital, Romford

- Referral returned to referring dentist because it was incomplete or inappropriate.

An evaluation was carried out using pluralistic methodologies to investigate management, patient and user (referring dentist) outcomes.

\section{Investigation of management outcomes}

Data were collected from the referral forms submitted by the referring dentists and from the invoices submitted by the DPwSIs. The information extracted from each referral form included referral date, referring dentist's name, reason for referral, clinical assessment date and outcome, and onward referral date. The information extracted from each invoice was contact date with patient and treatment provided. Management outcomes included the number of referrals received by CAS and outcome for each referral, the profile of the referred patient, the referral patterns of the referring dentists, the waiting time between referral and clinical assessment, the waiting time between referral and treatment, and costs comparison between treatment provided by DPwSI and secondary care.

\section{Investigation of patient outcomes}

Patient outcomes included satisfaction with the DPwSI, treatment received, and waiting time for treatment. Following receipts of invoices from the DPwSIs, the CAS manager carried out telephone interviews with a sample of randomly selected patients using a questionnaire guide that was agreed by the project team.

\section{Investigation of user outcomes}

User outcomes included the number of referring dentists who were aware of the service, the number who had used it, and their experiences. A questionnaire was mailed to all primary care dentists in December 2007. This was supported by a PCT headed letter from the consultant in dental public health to indicate PCT support for the evaluation. Respondents were assured that the information they volunteered would be treated confidentially. Stamped addressed envelopes were enclosed for return of completed questionnaires to the lead author's (AP's) office, which was geographically different to the PCT office in order to assure respondents that the information volunteered was not accessible to the PCT. A reminder letter and questionnaire was sent after a four-week interval and not sooner in order to avoid the Christmas holiday period and improve the response rate.

\section{RESULTS}

\section{Management outcomes}

During the three-month pilot period the CAS team received 83 referrals from 33 dentists. Just over half (57\%) of the referred patients were female. The mean age of the 83 referred patients was 44 years, with a range of seven to 85 years. Forty-one percent reported exemption from dental charges, 46\% no exemption, and 13\% did not respond.

Thirty of the referring dentists made one to four referrals each, whereas the three remaining dentists made over four referrals each, accounting for 29 (35\%) of all 83 referrals.

Of the 83 referrals, 64 (77\%) indicated extractions of wisdom teeth or special difficulty, whereas 19 (23\%) were referred for reasons related to complex medical history, patient anxiety, requests for sedation or general anaesthesia, or other oro-facial conditions. The 19 inappropriate referrals were made by 12 (36\%) of the dentists, with two dentists accounting for nine referrals. Of those who were inappropriately referred a higher proportion (53\%) was exempt from dental charges compared to those (appropriately) referred for extractions (38\%).

The date of referral or assessment was missing in 17 referral or outcome forms. It was therefore not possible to determine the assessment waiting time for these referrals. of the remainder 66 referrals, the number of days between referral and assessment was between one and 26, with a mean of seven. Nearly three-quarters of referrals were assessed within one week.

Following clinical assessment by CAS, $66(81 \%)$ referrals were directed to the DPwSIs, 12 (15\%) were directed to BHRT, 
three $(4 \%)$ were returned to the referring dentists, and two were incomplete (Fig. 1). Of the 66 referrals directed to the DPwSIs, $46(70 \%)$ were treated with extractions, five $(7 \%)$ received consultation and onward referral to BHRT, and 15 (23\%) were not contactable or did not attend their appointment.

Of the 51 patients (five for consultation and 46 for extractions) that were seen by a DPwSI during the pilot period, data on dates of treatment were available for 41 patients. The average waiting time between the referral date and the treatment date was 36 days, with a range of one to 98 days. Three quarters of those referred were seen within 43 days, or just over six weeks.

The cost of clinically assessing 83 referrals to the DPwSI-MOS and providing the necessary treatment to those who needed extractions during the three-month pilot period was $£ 8,020$, which included $£ 200$ for five cases of consultation (only) and $£ 7,820$ for 46 cases of extractions (Table 1). Treatment of these 46 cases at BHRT would amount to $£ 43,608$ - based on the tariff of $£ 948 /$ case charged by the hospital. This is a difference of $£ 35,588$ over a threemonth period and a projected difference of $£ 142,352$ for one year.

\section{Patient outcomes}

Eighteen patients were interviewed after attending for treatment by a DPwSI. Ten (57\%) reported that their referring dentists explained to them that they would be seen by a DPwSI-MOS based in a local dental practice and 12 (67\%) reported that they were able to choose a suitable appointment date, with 14 (78\%) reporting that they were seen on time. All patients reported that the DPwSIs explained the procedure, with 15 (88\%) reporting they were given instructions for after care and ten (57\%)

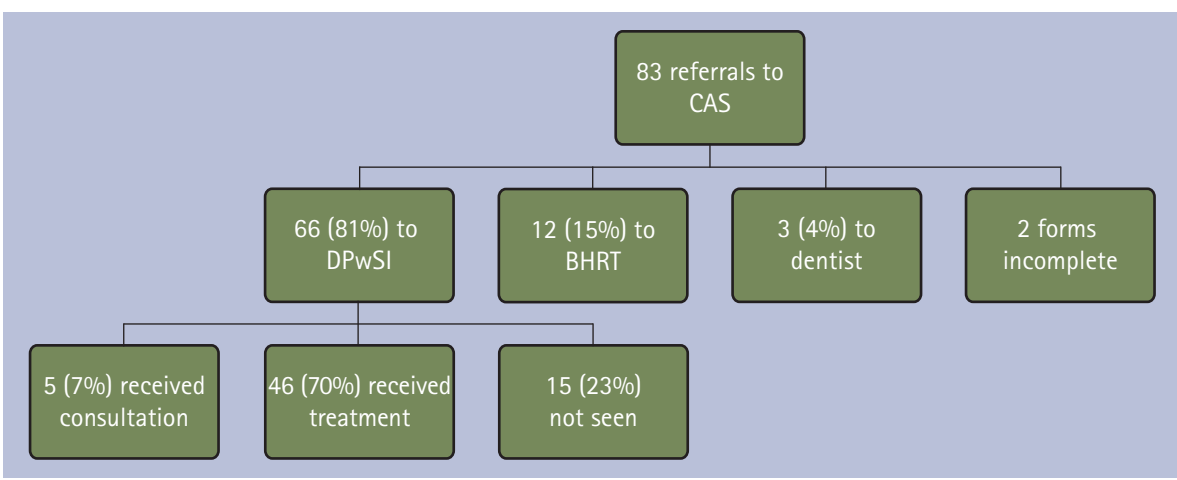

Fig. 1 Flow chart showing outcomes for patients referred to the DPwSI-MOS for extractions of wisdom teeth or special difficulty

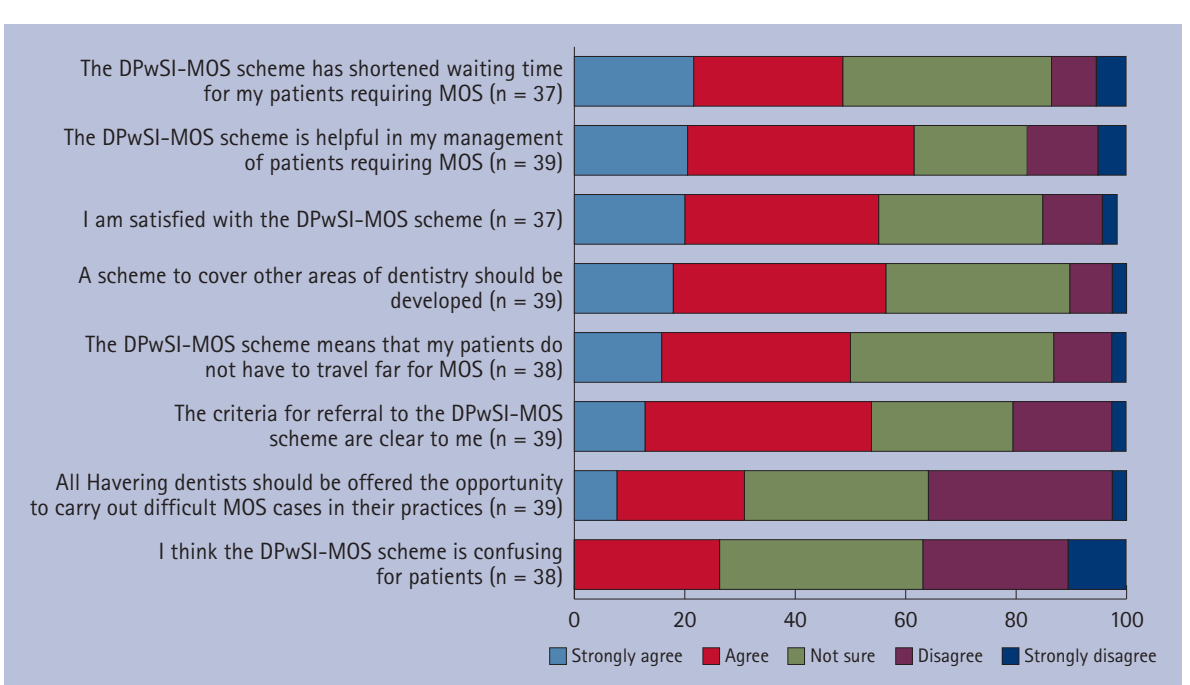

Fig. 2 Referring dentists' views of the DPwSI-MOS Scheme

reporting they were given an emergency telephone number to ring. Most patients reported that they were seen within two weeks (ten, 57\%) and four weeks (five, 28\%) from the time they were referred. Overall, the majority of the patients $(15,83 \%)$ rated the service as good to excellent.

\section{User outcomes}

Of the questionnaires mailed to all 90 dentists practising in Havering, 45 (50\%) were returned. Forty dentists (90\%) reported they were aware of the DPwSI, of whom, 24 $(60 \%)$ reported having used the service. Just over 50\% of those who had used the service reported referring on average one patient a month. The remaining dentists referred a range of two to four patients a month.

of those referring dentists who were aware of the DPwSI, 21 (53.8\%) reported that the criteria for referral were clear (Fig. 2). Although 24 (61.5\%) respondents reported that the service was helpful in their management of patients requiring MOS and 21 (55.1\%) reported that they were satisfied, around half were not sure if the service actually benefited their patients, with only 18 (48.6\%) suggesting

Table 1 Cost comparison between treatment under the DPwSI-MOS and treatment provided at BHRT

\begin{tabular}{|l|l|l|l|l|l|l|l|l|l|l|l} 
& $\begin{array}{l}\text { No. of } \\
\text { referrals } \\
\text { received }\end{array}$ & $\begin{array}{l}\text { No. } \\
\text { directed } \\
\text { to DPwSIs }\end{array}$ & $\begin{array}{l}\text { No. not } \\
\text { seen }\end{array}$ & $\begin{array}{l}\text { No. consulted } \\
\text { and referred } \\
\text { to BHRT }\end{array}$ & $\begin{array}{l}\text { Cost }(€) \text { per } \\
\text { non-surgical } \\
\text { consultation }\end{array}$ & $\begin{array}{l}\text { Cost (€) of } \\
\text { consultation } \\
\text { with surgery }\end{array}$ & $\begin{array}{l}\text { Number } \\
\text { treated by } \\
\text { DPwSls }\end{array}$ & $\begin{array}{l}\text { Cost (€) } \\
\text { per case } \\
\text { treated }\end{array}$ & $\begin{array}{l}\text { Cost }(€) \\
\text { of treat- } \\
\text { ment }\end{array}$ & $\begin{array}{l}\text { Total (€) } \\
\text { cost }\end{array}$ \\
\hline DPwSI-MOS & 83 & 66 & 15 & 5 & 40 & 200 & 46 & 170 & 7,820 & 8020 \\
\hline BHRT & & & & & & & $46^{* 1}$ & $948^{* 2}$ & 43,608 & 43,608 \\
\hline Cost difference & & & & & & & & & & 35,588 \\
\hline
\end{tabular}


that the waiting time for patients had shortened, and 19 (50.0\%) suggesting that their patients did not have to travel for MOS. Fourteen (36.8\%) dentists did not agree that the service was confusing for their patients, while the same proportion was not sure. A minority of the referring dentists (31\%) thought that they should be offered the opportunity to carry out difficult MOS cases in their practices. Just over half (56\%) thought that a similar service to cover other areas of dentistry should be developed.

When asked to comment on how the service could be improved, the following concerns were voiced:

1. Difficulty in tracking referrals

2. Difficulty in making referrals if a panoral radiograph was needed but the practice did not have such a facility

3. Patients referred for extractions may be persuaded by the DPwSIs to have other treatment.

\section{DISCUSSION}

This evaluation of DPwSI has demonstrated that locally accessible and acceptable MOS care that are cost-effective can be provided well within the recommended 18-week pathway. Other key findings of this evaluation are that a significant minority of referrals were inappropriate, not all primary care dentists were using the service, and a high proportion of those using the service were not sure of its benefits to their patients. The learning for the future development of this service in Havering PCT and other trusts planning to implement such a service is discussed.

The positive ratings by patients and referring dentists who used the DPwSI service provide an argument to support the provision of such a service. The benefits demonstrated by this evaluation included support for local dentists in their management of patients with MOS needs, management of the 18-week waiting time pathway, and efficient use of PCT resources. It may be argued that using dentists to provide special interest care may have the effect of reducing the capacity for primary general dental care. However, the opportunity arises for utilising dental care professionals to meet this capacity, leading to a quality workforce with the appropriate skills-mix.
Feedback from referring dentists suggested that the DPwSI should be further expanded to include other areas of minor oral surgery such as frenectomy, removal of simple cysts, and apicectomy. Furthermore, the learning from this pilot should be used to inform the development of DPwSI in other areas of dentistry such as treatment under sedation, endodontics and periodontics. Learning from the referrals audit would be particularly useful in developing the referral process in other DPwSI services. Development of the local workforce will be necessary to achieve this progress. This would involve training with appropriate consultants.

Nearly three-quarters of the patients were seen within six weeks of referral. Information on the specific waiting time for wisdom teeth extractions at BHRT was not available therefore comparison of waiting times was not possible. The cost difference when compared to treatment at BHRT was $£ 35,588$ for 46 patients over a three-month period. Extrapolating these figures to a period of a year would mean cost difference of $£ 142,352$. The costs saved would have been higher if all dentists had referred their patients requiring MOS extractions to the DPwSI. Results of the referrals audit and referring dentists' survey suggested that only a minority referred their patients to the service. The service was accessed by just over a third of all dentists during the pilot period. Postal questionnaire responses suggest that the reasons for this may be that some dentists did not have the need to use this service or that they did not perceive the service as benefiting their patients. The service is only sustainable if primary care dentists appreciate its benefits. A communications strategy is proposed to disseminate the findings of this evaluation as not all dentists were aware of its outcomes. The positive outcomes of this project should be communicated in order to encourage referring dentists to use this service for the benefits of their patients. This may be carried out through a 'service information leaflet' explaining the service and highlighting its positive aspects. Dentists should also be assured that the service is for specific MOS procedures, and that the DPwSIs will not retain patients referred to them. In addition, the referral patterns of individual dentists should be communicated back to them. Those with unusually high referral rates should be encouraged to examine their referral patterns and consider whether they are referring patients who need routine simple extractions. To ensure that this communication strategy is maintained, management information that is collected, such as information from the referral forms, should be systematically collated to allow continuous monitoring and auditing of the service.

Results of the referrals audit also indicated that not all referrals received by the CAS team were for extractions of wisdom teeth or special difficulty, thus incurring unnecessary workload on the CAS team as well as prolonging the referral time for those patients who should have been referred elsewhere. The referral protocol should therefore be redesigned to ensure that referrals are made for specific reasons, for example, by providing a tickbox for extractions of wisdom teeth or special difficulty in the referral form. This would ensure that the criteria for referrals are clearly stated on the referral form and prevent referrals for reasons other extractions of wisdom teeth and special difficulty such as patients with a compromised medical history, dentally anxious patients and patients requesting sedation or general anaesthesia. A reminder on the referral form to ensure referring dentists include patient contact information, especially telephone numbers, should be incorporated in the referral form. The referral protocol should be flexible to accommodate those dentists who do not possess certain facilities such as a panoral radiographic machine for taking radiographs of impacted wisdom teeth. The referral form should allow for a specific description of the indication for referral through a tick-box format. Although during the pilot phase the costs of clinical assessment were absorbed by the CAS, the cost element of assessing the referrals should be considered. Based on 83 referrals over three months, an equivalent of seven referrals per week, a clinical assessor for one hour a week at $£ 70$ an hour would amount to £3,640 per year. However, given that almost all appropriate referrals (as determined from the referral form) were referred on to the DPwSI-MOS after assessment by a clinician at CAS it may 
be argued that a clinical assessment is not necessary. Therefore if it is clearly indicated on the referral form that the patient needs an extraction of a wisdom tooth or of special difficulty, this referral may be forwarded directly to a DPwSI. Furthermore, the referral process may be streamlined by having clearly defined clinical guidelines to aid the assessment of referrals. This would also be facilitated by having an electronic referral system in which referrals and supporting radiographs may be sent electronically to make the process more efficient.

In summary, this evaluation of the first three months of the project has yielded the following learning for developing the project into a mainstream service:

- Re-design of the referral process to eliminate ambiguity and ensure greater precision

- Develop a communication strategy to share the outcomes of the project, especially its impact on the referring dentists' patients. The strategy should also allow for feeding back referral patterns to the referring dentists to highlight referral behaviours that deviate from the average

- Develop a structured process of obtaining patients' feedback in order to monitor their satisfaction with the service.

However, the findings from this evaluation should be considered in the context of its limitations. This evaluation has captured the views of a sample of patients and referring dentists at one point in time in Havering PCT. Future research is needed to determine the longer term effects of this service. In particular, before widespread implementation of MOS DPwSI schemes, a randomised controlled trial should be carried out to compare the outcomes for patients treated by DPwSI in the primary care setting and those treated in the secondary care setting.

\section{CONCLUSIONS}

The findings of this evaluation have demonstrated that there are potential benefits in the provision of MOS in the primary care setting by DPwSIs. These include satisfaction with access for patients, support for patient management for referring dentists and cost savings for the PCT. However, longitudinal evaluation is necessary to confirm the long term effects of this service.

1. National Health Service Act 1977. Primary Care Trust Dental Services Directions, 2006.

2. Department of Health. Commissioning NHS primary care dental services: meeting the NHS operating framework objectives. London: DH Publications, 2008.

3. Department of Health. NHS Plan. London: DH Publications, 2000.

4. Department of Health. Guidelines for the appointment of Dentists with Special Interests (DwSIs) in minor oral surgery. London: DH Publications, 2006.

5. Department of Health. The NHS Improvement Plan: putting people at the heart of public services. London: DH Publications, 2004.

6. London Health Observatory. Top ten procedures: DSRs by PCT and crude rates by hospital trust. Verified November 2008. http://www.lho.org.uk/ viewResource.aspx?id=11623 2007 .

7. Drake S. Dentists with special interests - towards a flexible workforce to meet oral health needs of the future. Br Dent J 2008; 205(5 Suppl): 9-11. 